\title{
Development of OLED Display Technology Based on Bibliometrics
}

\author{
Xuan Liu ${ }^{1, a}$, Jin Huang ${ }^{2, b,{ }^{*}}$, Chang Liu ${ }^{3}$, Fengping Wei ${ }^{4}$, Wenyan Liu ${ }^{5}$, \\ Xiaohong $\mathrm{Li}^{6}$, Changyan Yan ${ }^{7}$ \\ 1,2,3,4,5,6,7 Library of Huazhong University of Science and Technology Wuhan, Hubei, China \\ a liuxuan@hust.edu.cn, b hjhust@hust.edu.cn
}

\begin{abstract}
Keywords: Bibliometrics; OLED display technology; Optics Valley; visual analysis;
\end{abstract}
\begin{abstract}
This paper focuses on OLED display technology and uses bibliometric methods to first analyze the overall situation of scientific papers in the world and in China's industrial fields. Then take Hubei Optics Valley as an example to analyze Hubei Province's resources in this field. The configuration and the use of Citespace software to analyze the research hot spots of relevant scientific papers in this field will help to find out the focus of universities in enhancing scientific and technological innovation capabilities and provide support services for industrial innovation.
\end{abstract}

\section{Introduction}

The organic light-emitting diode (OLED) was discovered in the laboratory in 1979. OLED display technology has advantages such as self-luminescence, wide viewing angle, almost infinite contrast, lower power consumption, and extremely high reaction speed. The display technology is widely used in mobile phones, digital video cameras, DVD players, personal digital assistants (PDAs), notebook computers, car audio and television, and has a wide range of application prospects ${ }^{[1]}$. Therefore, this field has become one of the important research topics.

All countries and regions in the world use solid-state lighting as a technology that "illuminates the future." They have formulated a national plan for the development of solid-state lighting technology. For example, the United States has formulated the National Semiconductor Lighting Research Program, Japan has established the 21st Century Lighting Plan. Optics Valley, as the main force participating in the global optoelectronics industry competition on behalf of the country, is located in Wuhan, Hubei Province, China's largest optical communications research and development base, is China's largest fiber optic cable production base.

The form of scientific research results is mainly academic papers. By studying the various indicators, contents, and mutual reference characteristics of the papers, it is possible to objectively reveal the mutual penetration between science and technology and between basic research and technological innovation. Impact and promotion ${ }^{[2]}$. From the perspective of bibliometrics, this article uses the Web of Science platform, the Chinese CNKI platform and the EI Compendex database to conduct relevant searches on this field of published papers, and uses the knowledge mapping tools to research hotspots in Hubei Province in this research field.

\section{Overall Performance of OLED Data}

\subsection{International and China's data}

The Web of Science platform: The Web of Science information retrieval platform can retrieve high-quality literature on the natural sciences, social sciences, arts and humanities. Search strategy: Subject search in the WOS core collection with "Organic Light Emitting Diode" OR "OLED" OR "Organic Electro Laser Display" OR "Organic Light Emitting Semiconductor" covering a time range from 2007 to 2017 for a total of 10 years. The search scope is defined as follows: SCI-EXPANDED, SSCI, A\&HCI, CPCI-S, CPCI-SSH, and data type are defined as article, review, and proceedings papers. A total of 9,392 articles have been retrieved, of which 1915 articles have been published in Mainland China, accounting for nearly $20 \%$ of the total. It can be seen that the 
main researchers in this field are universities. The Chinese Academy of Sciences has the largest output and ranks first in the world.

CNKI Academic Resource Center: Subject search using ('Organic Light Emitting Diode' + 'OLED' + 'Organic Laser Display' + 'Organic Light Emitting Semiconductor') as the search query, from 2007 to 2017, and get total of 5284 articles. The scope of the discipline of published papers is mainly distributed in the fields of radio electronics, industrial economy, telecommunication technology, and chemistry. It can be seen that the most published institutions of OLED-related Chinese papers are universities except for the Changchun Institute of Optics and Mechanics, among which Jilin University, University of Electronic Science and Technology and South China University of Technology are among the top three.

\subsection{Hubei Regional Research on OLED Technology}

In order to further study the promoting effect of this technology on the regional economy, we use ((oled or "Organic Light-Emitting Diode") WN KY) AND ((wuhan or hubei or 4300* same univ*) WN AF in the EI Compendex database. )) to search, a total of 332 articles. Among them, the National Key Laboratory of Optoelectronics of Huazhong University of Science and Technology and the Wuhan University have performed outstandingly.

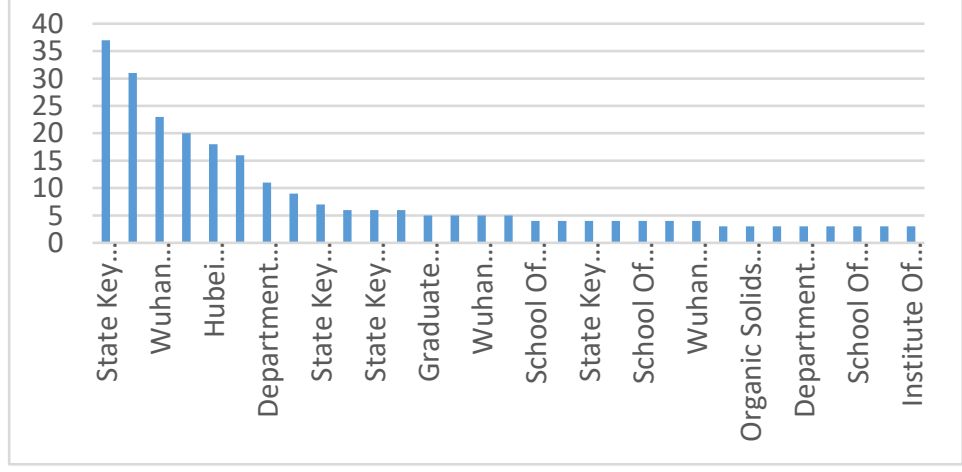

Figure.1. OLED Hubei Research Power Distribution

Then, in the wos, we searched the achievements of oled in Wuhan or Hubei universities. A total of 66 records were detected. The search structure is: Subject: (oled or "Organic Light-Emitting Diode") AND Address: (wuhan or hubei or 4300* same univ*). It can be seen that Wuhan University, Huazhong University of Science and Technology, Hubei University of Technology, and Hubei University all have relevant research. Wuhan University has performed well, with Wuhan University and Huazhong University of Science and Technology accounting for nearly $70 \%$. Most of the time is distributed in 2000-2017.

\section{Clustering and Hotspot Analysis of OLED Research in Hubei Region}

In order to further study the status of OLED in Hubei and explore the role of scientific and technological achievements in guiding and serving regional economies, this paper uses Citespace software for analysis. The software traces and analyzes the sudden changes (increase) in the frequency of terminology in different time intervals and identifies several terminology terms that represent the research frontier. Since this study does not focus on discovering the "evolution" process of hotspots, but focuses on the hot research content of the entire time period, only one time zone is divided in the Citespace interface ${ }^{[3]}$. The time span spans 10 years (2007-2017), and the node type Select "Cited Reference" and "Burst Term", using the Stanford NLP Group Part-of-speech tagging technology, extracting terms from Title, Abstract, Author Keywords (DE) and Keyword Plus (ID), and further generating co-words The internet. After the analysis results are visualized, the co-citation network is clustered, the clustered labels are extracted from the titles of the citing articles, and the tag word extraction method is selected as $\mathrm{tf}^{*} \mathrm{df}$. 
After analyzing the Keyword extracted from these documents and using software visualization, it can be seen that the keyword of oled research in Hubei or Wuhan

Cluster keywords, one \# represents a cluster. Based on the network structure and the clarity of the clustering, Citespace provides two indicators of module value ( $Q$ value) and average contour value ( $\mathrm{S}$ value) to evaluate the clustering effect. When $\mathrm{Q}>0.3$, it means that the obtained network community structure is significant, and $\mathrm{S}>0.5$ can be considered as a reasonable clustering result.

The $Q$ value of this clustering is 0.5942 , and the $S$ value is 0.8293 , which shows that the characteristics of the literature clusters are obvious, the clustering results have high reliability, and the maps have good results. The nodes in Figure 4 include two types of citations and mutated words.

Citespace's clusters have found nine clusters (the labels of each cluster are numbered by "\#" and Arabic numerals). According to the cluster labels and mutations of various clusters and the content of key nodes within clusters, four research hotspots in the OLED field can be summarized. ${ }^{[4]}$

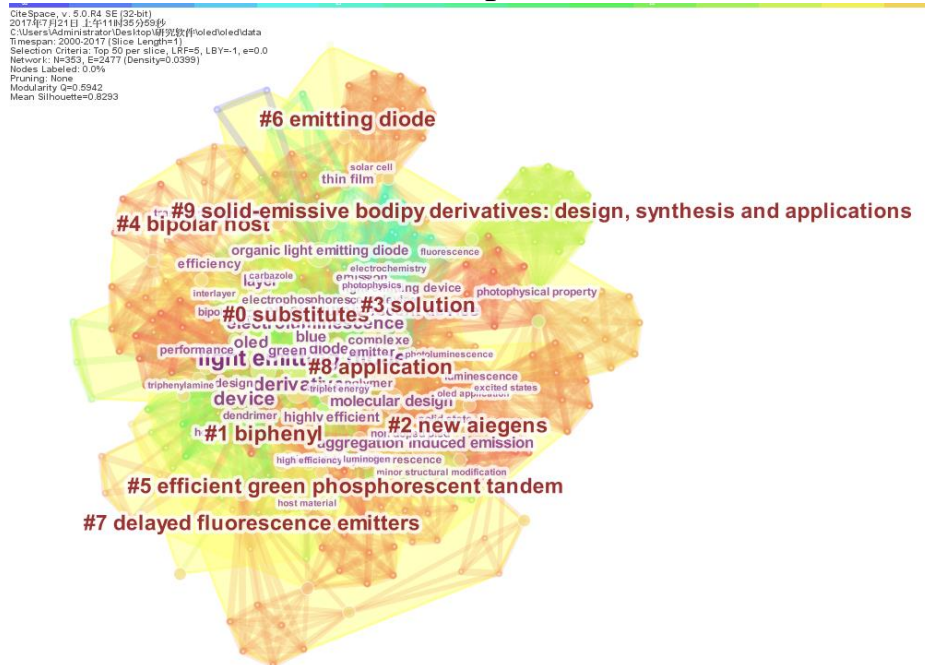

Figure.2. 3.Clustering and Hotspot Analysis

(1) Research on the principles and materials of OLED based on LED related research. Topics include photophysics, iridium complexes, and dendrimers.

(2) Research on OLED luminescence (electroluminescence, photoluminescence) and new OLED preparation materials represented by ITO, PPV and derivatives and PAT. Among the hot topics are polymers, electrochemistry, thin film transistors.

(3) Research on organic photovoltaic solar energy materials and technologies. Include OLED optical properties, carrier injection and transport and recombination, doping, conjugated polymers, and excitons.

(4) Preparation of materials, methods and luminescence principles for polymer OLEDs. Among the hot topics are self-assembly, carbazole, energy transfer and electrical structure.

\section{References}

[1] Chen Weihua, Research progress in the interface of organic light-emitting devices[J]. Journal of Taiyuan University of Technology, issue3,pp.350-361, 2017

[2] Wang q, MA D. Management of charges and excitons for high-performance wihte organinc light-emitting diodes[J]. Chemical Society Reviews, vol. 39, issue7,pp.2387-98 1, 2010

[3] Brod M, Tesler L E, Christensen T L. Qualitative research and content validity: developing best practices based on science and experience[J]. Quality of Life Research. vol.18, issue9,pp. 1263-78, 2009

[4] MAH,YIPH,Huang F,Interface egnieering for organic electronics[J]. Advanced functioal materials, vol.20, issue9,pp.1371-88, 2010 
